\title{
Surgical treatment for obstructive hypertrophic cardiomyopathy
}

\author{
Kenji Minakata $\cdot$ Ryuzo Sakata
}

Received: 19 April 2013/Accepted: 14 May 2013/Published online: 28 May 2013

(C) The Japanese Association for Thoracic Surgery 2013

Keywords Hypertrophic cardiomyopathy $\cdot$ Mitral valve replacement $\cdot$ Septal myectomy

Hypertrophic cardiomyopathy (HCM) is one of the most common genetic diseases affecting myocardium with wide variety of clinical manifestation, hemodynamic characteristics, anatomic morphology, and natural history. Patients with HCM may develop limiting symptoms due to dynamic left ventricular outflow tract obstruction (LVOTO) and associated mitral regurgitation (MR) due to systolic anterior motion (SAM) of the anterior mitral leaflet [1]. For those who have severe symptoms unresponsive to maximum medical therapy including beta-blockers and antiarrhythmic drugs such as disopyramide or cibenzoline, surgical left ventricular septal myectomy has been the standard option. It has been shown that septal myectomy significantly decreases LVOT gradient and associated MR and provides excellent long-term outcomes.

Although the basic transaortic approach for performing a septal myectomy has been known for over 40 years, the operation remains technically challenging and results are operator dependent to a high degree [2]. This procedure carries many tips and pitfalls. Incisions or excisions made too deep may create ventricular septal defects or ventricular perforations, both of which increase surgical mortality significantly. Poor visualization of the anatomy below the

This comment refers to the article available at doi:10.1007/s11748-013-0245-1.

K. Minakata $(\varangle) \cdot$ R. Sakata

Department of Cardiovascular Surgery, Kyoto University

Graduate School of Medicine, 54 Kawahara-cho, Shogoin,

Sakyo-ku, Kyoto 606-8507, Japan

e-mail: minakata@kuhp.kyoto-u.ac.jp aortic valve can result in injury to mitral leaflets or chordae. Incorrect placement of incisions or excessive traction can produce complete heart block, requiring permanent pacemakers. Inadequate myocardial protection of the hypertrophied heart can cause difficulty in defibrillation and low cardiac output, which may require mechanical circulatory support. The aortic valve is always at risk for injury from instruments passed through the valve and manipulated within the ventricle. Therefore, it is understandable that overly conservative surgical attitudes may sometimes prevail, resulting in limited myocardial resection and consequent incomplete relief or early return of LVOTO and symptoms. Even if adequate resection is surgically obtained at the level of the basal septum, midventricular obstruction and/or MR associated with abnormal papillary muscles and/or chordae, and other muscle bundles may persist, and additional procedures may become necessary [3].

As Furukawa et al. [4] reported their surgical case in this issue of the GTCS, in which mitral valve replacement (MVR) was required because of residual LVOTO and MR after standard septal myectomy, surgeons performing this procedure should always think about options for residual LVOTO and MR. Because SAM plays an essential role for LVOTO and MR, additional procedures such as mitral valve plasty including Alfieri's stitch or MVR with lowprofile prosthesis can be effective alternatives. It was first reported by Cooley et al. that MVR using mechanical valves eliminate MR and can relief LVOTO and symptoms in patients with obstructive HCM [5]. Because MVR is a simple and highly reproducible procedure, it can be an attractive option for those who have residual LVOTO and MR even after standard septal myectomy although those undergoing MVR with mechanical valve require life-long anticoagulation therapy. 


\section{References}

1. Maron BJ. Hypertrophic cardiomyopathy. Lancet. 1997;350: 127-33.

2. Minakata K, Dearani JA, Schaff HV, O'Leary PW, Ommen SR, Danielson GK. Mechanisms for recurrent left ventricular outflow tract obstruction after septal myectomy for obstructive hypertrophic cardiomyopathy. Ann Thorac Surg. 2005;80:851-6.

3. Minakata K, Dearani JA, Nishimura RA, Maron BJ, Danielson GK. Extended septal myectomy for hypertrophic cardiomyopathy with anomalous mitral papillary muscles or chordae. J Thorac Cardiovasc Surg. 2004;127:481-9.

4. Furukawa K, Hayase T, Yano M. Mitral valve replacement and septal myectomy for hypertrophic obstructive cardiomyopathy. Gen Thorac Cardiovasc Surg. 2013 (in-press).

5. Krajcer Z, Leachman RD, Cooley DA, Coronado R. Septal myotomy-myomectomy versus mitral valve replacement in hypertrophic cardiomyopathy. Ten-year follow-up in 185 patients. Circulation. 1989;80:I57-64. 